EUROPHYSICS LETTERS

1 December 1999

Europhys. Lett., 48 (5), pp. 486-490 (1999)

\title{
New integral relaxation time for thermal activation of magnetic particles
}

\author{
D. A. GARAnin ${ }^{1}$ (甘) \\ 1 Max-Planck-Institut für Physik komplexer Systeme, Nöthnitzer Str. 38, D-01187 Dres- \\ den, Germany
}

(received 10 May 1999; accepted 28 September 1999)

PACS. 05.40.-a - Fluctuation phenomena, random processes, noise, and Brownian motion. PACS. 75.50.Tt - Fine-particle systems.

\begin{abstract}
The integral relaxation time for the difference of numbers of particles in the two potential wells (IRT- $N$ ) for double-well classical spin systems is introduced. For the uniaxial symmetry, it is given by a quadrature. Unlike the previously introduced integral relaxation time for the magnetization, the IRT- $N$ at low temperatures describes the rate of crossing the barrier under all conditions, including the strongly biased case. In the high-barrier case, the new integral relaxation time approaches the inverse of the lowest eigenvalue of the Fokker-Planck equation $\Lambda_{1}$. It can be more conveniently found by numerical methods than the latter.
\end{abstract}

The rate of thermal activation $\Gamma$ of a dynamical system over a potential barrier $\Delta U$ between two wells is exponentially small in the low-temperature or high-barrier limit: $\Gamma=$ $\Gamma_{0} \exp (-\Delta U / T)$. In this limit, all definitions of the rate yield the same result. Analytical methods based on solving the Fokker-Planck equation (FPE) for the flow of the probability current over the barrier ware developed by Kramers [1] for the particles (see ref. [2] for a review) and by Brown [3] for magnetic particles (classical spins). In situations where analytical solution is problematic, the thermal activation rate $\Gamma$ was usually identified with the lowest eigenvalue $\Lambda_{1}$ of the Sturm-Liouville equation corresponding to the FPE and $\Lambda_{1}$ was calculated numerically [4]. In the high-barrier case, $\Lambda_{1}$ is exponentially small and describes the slow process of the exchange of particles between the two wells, whereas all other $\Lambda_{i}, i=2,3, \ldots$. are not exponentially small and correspond to intrawell processes. For spin problems in the high-temperature limit $\Delta U / T \ll 1$, the lowest eigenvalue $\Lambda_{1}$ describes relaxation of the magnetization and it is, again, more important than other eigenvalues describing relaxation of higher moments of the distribution function. At intermediate temperatures $\Delta U / T \sim 1$, the exclusive role of $\Lambda_{1}$ in the overbarrier relaxation is no longer obvious, since other eigenvalues are of the same order as $\Lambda_{1}$ and they can make contribution to this process, too. In addition, $\Lambda_{1}$ cannot be calculated analytically in this temperature range even for the simplest models, such as that of uniaxially anisotropic spins with or without a longitudinal field.

In fact, at intermediate temperatures the overbarrier escape rate is not unambiguously defined, and one can use various alternative definitions of $\Gamma$. One of the quantities measuring the escape rate is the mean first-passage time (MFPT) [5, 6] which for the simplest models can be calculated analytically in the whole range of temperatures. Another useful quantity which

$\left(^{*}\right)$ www.mpipks-dresden.mpg.de/ garanin/; garanin@mpipks-dresden.mpg.de

Typeset using EURO-TEX 
is more appealing to physicists is the integral relaxation time (IRT) $\tau_{\text {int }}$ which is defined by the area under the relaxation curve for the magnetization or polarization after an infinitesimal instantaneous change of the applied field [7, 8]. Equivalently, the IRT is given by the coefficient in the linear term in the low-frequency expansion of the susceptibility. For the uniaxial spins in a longitudinal field, the low-frequency perturbation theory for the FPE leads to the analytical solution for the IRT in the whole temperature range. For more complicated models, such as a uniaxial spin in an oblique field, the low-frequency susceptibility can be obtained numerically from the matrix-continued-fraction solution of the FPE [9]. This routine is much more convenient than diagonalization of large matrices to obtain $\Lambda_{1}$.

Numerical solutions for the $\tau_{\text {int }}$ and $\Lambda_{1}$ performed in ref. [9] for the uniaxial spin model in a longitudinal field revealed a striking feature. Whereas in zero field $\tau_{\text {int }}^{-1}$ coincides with $\Lambda_{1}$ in the whole temperature range to within only $1.2 \%$, both quantities drastically deviate from each other at low temperatures for the fields exceeding some critical value. Whereas $\Lambda_{1}$ is exponentially small, as it should be, $\tau_{\text {int }}^{-1}$ in not exponentially small and increases with the increase of the strength of the potential relief. The physical explanation of this effect was given in ref. [10]. In the biased low-temperature case, almost all particles or spins concentrate in the lower well, whereas the upper well is depleted. Then $\tau_{\text {int }}$ captures the fast relaxation in the lower well (the intrawell process) rather than the slow overbarrier relaxation process. Since the intrawell process is not what one is usually interested in, the results of refs. [9, 10] put the usefulness of the concept of the integral relaxation time in a general case under question.

The aim of this Letter is to introduce another type of the IRT which describes the process of the overbarrier relaxation in all situations. The key idea is to consider the responce of the population difference $\Delta \equiv N_{+}-N_{-}$, where $N_{ \pm}$are the numbers of particles in the two wells, to the changing bias field. Unlike the magnetization response used to calculate the IRT, the $\Delta$ response is insensitive to the redistribution of spins within the wells and always describes the overbarrier process. To emphasize the difference, it is convenient to designate the old (magnetization) IRT as IRT- $M$ and the new (population) IRT as IRT- $N$.

Let us consider a single-domain magnetic particle with a classical Hamiltonian

$$
\beta \mathcal{H}=v(\mathbf{n})-\mathbf{n} \cdot \delta \boldsymbol{\xi}(t, \omega), \quad \delta \boldsymbol{\xi}(t, \omega)=\delta \boldsymbol{\xi}_{0} \exp (-i \omega t),
$$

where $\beta \equiv 1 / T, \mathbf{n}$ is the unit vector representing the direction of the particle's magnetization, $\delta \boldsymbol{\xi}(t, \omega) \equiv \beta V M_{s} \delta H(t, \omega)$ is the reduced alternating field acting on the particle, $V$ is the particle's volume and $M_{s}$ is the saturation magnetization. The all-temperature analytical solution for the integral relaxation time is possible if $v(\mathbf{n})$ is axially symmetric and the alternating field is applied along the $z$ axis. The mostly studied model is a uniaxial particle in the longitudinal field

$$
v(x)=-\xi x-\sigma x^{2}, \quad x \equiv \cos \theta
$$

with

$$
\xi \equiv \beta V M_{s} H, \quad \sigma \equiv \beta V M_{s}^{2} K,
$$

where $K>0$ is the anisotropy constant creating a potential relief with two wells separated by a barrier. The top of the barrier corresponds to

$$
x=x_{m}=-h, \quad h \equiv \xi /(2 \sigma)=H /\left(2 K M_{s}\right),
$$

the barrier height $\Delta v \equiv v\left(x_{m}\right)-v(-1)$ is given by

$$
\Delta v=\sigma-\xi+\xi^{2} /(4 \sigma)=\sigma(1-h)^{2},
$$


for $h \geq 1$ the barrier disappears.

The dynamics of the classical single-domain magnetic particle is governed by the FokkerPlanck equation which was first formulated by Brown [3]. This FPE can also be found in refs. [8, 10, 11]. The equilibrium distribution function satisfying the static FPE can for axially symmetric models be written in the form

$$
f_{0}(x)=\frac{\exp [-v(x)]}{Z}, \quad Z=\int_{-1}^{1} \exp [-v(x)] \mathrm{d} x .
$$

If an infinitesimally small alternating longitudinal field is applied, the distribution function can be searched in the form

$$
f(x, t, \omega)=f_{0}(x)[1+q(x) \delta \xi(t, \omega)]
$$

where the complex function $q(x)$ satisfies the equation 8,10

$$
\left(\frac{\mathrm{d}}{\mathrm{d} x}-\frac{\mathrm{d} v}{\mathrm{~d} x}\right)\left(1-x^{2}\right) \frac{\mathrm{d} q}{\mathrm{~d} x}+2 i \omega \tau_{N} q=-\left(1-x^{2}\right) \frac{\mathrm{d} v}{\mathrm{~d} x}-2 x .
$$

Here $\tau_{N}$ is the Néel relaxation time defined by

$$
\tau_{N}^{-1} \equiv \Lambda_{N} \equiv 2 \gamma a T /\left(V M_{s}\right)
$$

where $\gamma$ is the gyromagnetic ratio and $a$ is the dimensionless Gilbert's damping parameter. The solution of eq. (8) should satisfy the normalization condition $\int_{-1}^{1} f_{0}(x) q(x) \mathrm{d} x=0$. The low-frequency expansion of $q(x)$ has the form

$$
\begin{aligned}
& q(x)=q_{0}(x)+i \omega \tau_{N} q_{1}(x)+\ldots, \quad q_{0}(x)=x-B \\
& q_{1}(x)=\int_{-1}^{x} p\left(x^{\prime}\right) \mathrm{d} x^{\prime}-\int_{-1}^{1} f_{0}(x) \int_{-1}^{x} p\left(x^{\prime}\right) \mathrm{d} x^{\prime} \mathrm{d} x,
\end{aligned}
$$

where $B=\int_{-1}^{1} x f_{0}(x) \mathrm{d} x$ is the equilibrium spin polarization and

$$
p(x)=\frac{2}{1-x^{2}} \frac{\Phi(x)}{f_{0}(x)}, \quad \Phi(x)=\int_{-1}^{x}\left(B-x^{\prime}\right) f_{0}\left(x^{\prime}\right) \mathrm{d} x^{\prime} .
$$

Having solved the FPE, one can calculate the dynamic susceptibility

$$
\tilde{\chi}(\omega)=\frac{1}{M_{s}} \frac{\delta M(t, \omega)}{\delta \xi(t, \omega)}=\int_{-1}^{1} x f_{0}(x) q(x) \mathrm{d} x \cong \tilde{\chi}\left(1+i \omega \tau_{\mathrm{int}}+\ldots\right), \quad \tilde{\chi}=B^{\prime} \equiv \frac{\partial B}{\partial \xi} .
$$

Using eq. (10) and integrating by parts one obtains the magnetization IRT [0, 8, 10

$$
\tau_{\text {int }}=\frac{2 \tau_{N}}{B^{\prime}} \int_{-1}^{1} \frac{\mathrm{d} x}{1-x^{2}} \frac{\Phi^{2}(x)}{f_{0}(x)} .
$$

In ref. 10 it was shown that in the biased case $\xi>0$ at low temperatures the integrand of this formula peaks near the top of the barrier $x=x_{m}$ and in the lower well $x \approx 1$, which corresponds to the overbarrier and intrawell processes, respectively. For $h>h_{c}=3-2 \sqrt{2} \approx 0.17$ at low temperatures, the upper well is depleted, the overbarrier process is thus suppressed, and the intrawell process controls the IRT. This undesirable feature makes one to look for a more appropriate definition of the integral relaxation time. 


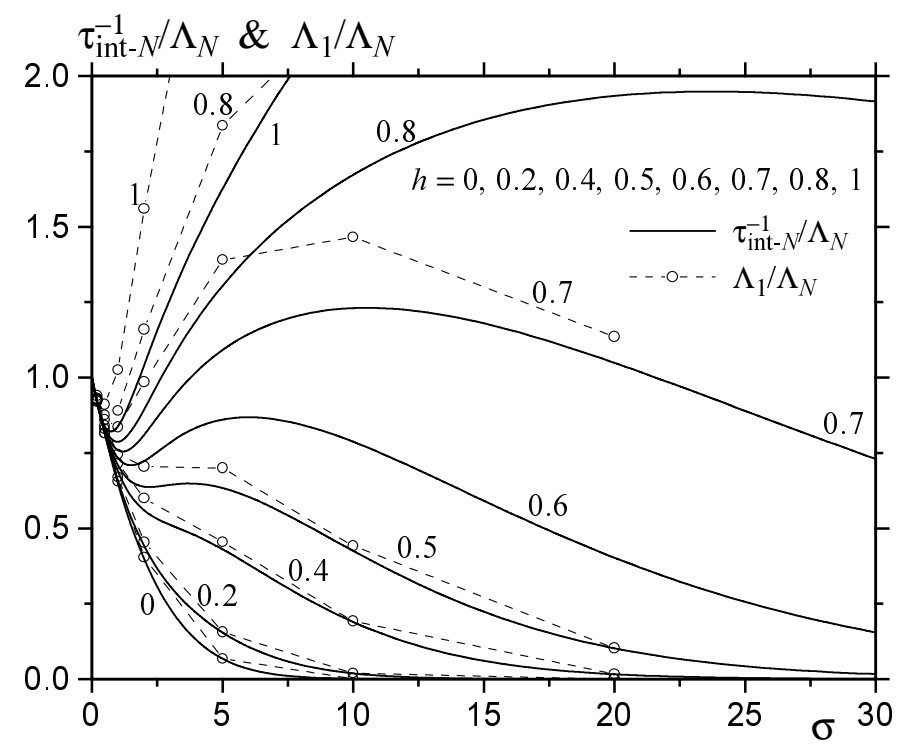

Fig. 1. - Inverse of the integral relaxation time for populations IRT- $N$, eq. (18), and the lowest eigenvalue of the Fokker-Planck equation $\Lambda_{1}[9]$ vs the potential strength $\sigma$.

The simplest choice for the quantity which is insensitive to the intrawell process is the difference of the wells populations

$$
\Delta(t, \omega) \equiv N_{+}-N_{-}=\int_{-1}^{1} \operatorname{sgn}\left(x-x_{m}\right) f(x) \mathrm{d} x .
$$

In equilibrium one has

$$
\Delta=\frac{Z_{+}-Z_{-}}{Z_{+}+Z_{-}}, \quad Z_{-}=\int_{-1}^{x_{m}} f_{0}(x) \mathrm{d} x, \quad Z_{+}=\int_{x_{m}}^{1} f_{0}(x) \mathrm{d} x,
$$

where $Z_{ \pm}$are the contributions to the partition function $Z$ from both wells. The dynamic susceptibility for the population difference $\Delta$ has the form

$$
\tilde{\chi}_{\Delta}(\omega)=\frac{\delta \Delta(t, \omega)}{\delta \xi(t, \omega)}=\int_{-1}^{1} \operatorname{sgn}\left(x-x_{m}\right) f_{0}(x) q(x) \mathrm{d} x \cong \tilde{\chi}_{\Delta}\left(1+i \omega \tau_{\mathrm{int}, N}+\ldots\right),
$$

where

$$
\tilde{\chi}_{\Delta}=\Delta^{\prime} \equiv \frac{\partial \Delta}{\partial \xi}=\frac{2\left(Z_{-} Z_{+}^{\prime}-Z_{+} Z_{-}^{\prime}\right)}{\left(Z_{+}+Z_{-}\right)^{2}}, \quad Z_{ \pm}^{\prime} \equiv \frac{\partial Z_{ \pm}}{\partial \xi},
$$

and the integral relaxation time for the populations is given by

$$
\tau_{\text {int }, N}=\frac{2 \tau_{N}}{\Delta^{\prime}} \int_{-1}^{1} \frac{\mathrm{d} x}{1-x^{2}} \frac{\Phi_{N}(x) \Phi(x)}{f_{0}(x)}, \quad \Phi_{N}(x)=\int_{-1}^{x}\left[\Delta-\operatorname{sgn}\left(x^{\prime}-x_{m}\right)\right] f_{0}\left(x^{\prime}\right) \mathrm{d} x^{\prime} .
$$

This result follows from the same low-frequency solution of the FPE, eq. (10). It can be checked that the function $\Phi_{N}(x)$ has no maxima near the bottom of the wells, thus $\tau_{\text {int }, N}$ does not 
describe the intrawell process. This new integral relaxation time, which can be abbreviated as IRT- $N$, captures the overbarrier process in the low-temperature range for all values of the longitudinal field.

Dependences of $\tau_{\text {int, } N}^{-1}$ on the barrier-strength parameter $\sigma$ for different values of $h$ for the uniaxial model [see eqs. (2) and (3)] are shown in Fig. 1, compared to the values of the lowest eigenvalue $\Lambda_{1}$ quoted in Table I of ref. [9]. One can see that both quantities behave similarly, in contrast to $\tau_{\text {int }}^{-1}$ (cf. Figs. 1 and 2 of ref. [9]). In the region where the escape rate becomes exponentially small, $\tau_{\text {int, } N}^{-1}$ and $\Lambda_{1}$ coincide with each other. An interesting feature of the dependences in Fig. 1 is the maximum of the relaxation rate arising in the strongly biased case, which is more pronounced for $\Lambda_{1}$ than for $\tau_{\text {int, } N}^{-1}$. The physical reason for this maximum is the following. As long as the parameters $\sigma$ and $\xi$ are large but the reduced barrier height $\Delta v=\sigma(1-h)^{2}$ is small, the dynamics of the magnetic particle is qualitatively the same as that of a magnetic particle without a potential barrier at low temperatures. In this case the relaxation rate can be found in a simple way from the deterministic Landau-Lifshitz equation and it is proportional to the strength of the potential relief, i.e., to $\sigma$. If $\sigma$ is further increased, then for any $h<1$ the reduced barrier hight $\Delta v$ becomes large for $\sigma \gg 1 /(1-h)^{2}$, and the escape rate becomes exponentially small. These two regimes are separated by a maximum of the relaxation rate.

In this Letter, the integral relaxation time for populations, IRT- $N$, was introduced. This quantity is more useful than the previously considered integral relaxation time for the magnetization, IRT- $M$, and it always captures the exponentially slow process of thermal activation in the high-barrier limit. For the simplest models considered here, there is an all-temperature solution for the IRT- $N$ in quadratures. In other cases, the problem can be solved numerically considering the low-frequency limit of the matrix-continued-fraction solution of the FPE [9]. Formulation of the method for non-axially symmetric potentials or for (mechanical) particles poses no problems. Numerical calculation of the IRT- $N$ is a more convenient and accurate procedure than that for the lowest eigenvalue $\Lambda_{1}$. Using the new integral relaxation time should help to throw a light on fine details of various crossovers which have been recently discovered for the uniaxial model in a transverse field [1]. For this model, numerical calculation of $\Lambda_{1}$ faces difficulties in the low-damping high-barrier case.

The author thanks Oleg Yevtushenko for critical reading of the manuscript.

\section{REFERENCES}

[1] H. A. Kramers, Physica 7, 284 (1940).

[2] P. Hänggi, P. Talkner, and M. Borkovec, Rev. Mod. Phys. 62, 251 (1990).

[3] W. F. Brown, Jr., Phys. Rev. 130, 1677 (1963); IEEE Trans. Magn. MAG-15, 1196 (1979).

[4] A. Aharoni, Phys. Rev. 135A, 447 (1964); 177, 793 (1969).

[5] G. Klein, Proc. R. Soc. London A 211, 431 (1952).

[6] H. Risken, The Fokker-Planck Equation (Springer, Berlin, Second Edition, 1989).

[7] A. Szabo, J. Chem. Phys. 72, 4620 (1980).

[8] D. A. Garanin, V. V. Ishchenko, and L. V. Panina, Teor. Mat. Fiz. 82, 242 (1990).

[9] W. T. Coffey, D. S. F. Crothers, Yu. P. Kalmykov, and J. T. Waldron, Phys. Rev. B 51, 15947 (1995).

[10] D. A. Garanin, Phys. Rev. E 54, 3250 (1996).

[11] D. A. Garanin, E. Kennedy, D. S. F. Crothers, and W. T. Coffey, Phys. Rev. E 60, 6499 (1999). 